\title{
Light signals from a lighter Higgs
}

\section{Patrick J. Fox ${ }^{a}$ and Neal Weiner ${ }^{b, c}$}

a Theoretical Physics Department, Fermilab, Batavia, IL 60510, U.S.A.

${ }^{b}$ Center for Cosmology and Particle Physics, Department of Physics,

New York University, New York, NY 10003

${ }^{c}$ Center for Computational Astrophysics, Flatiron Institute, 162 Fifth Ave, New York, NY 10010, U.S.A.

E-mail: pjfox@fnal.gov, neal.weiner@nyu.edu

ABSTRACT: With the Higgs search program already quite mature, there is the exciting possibility of discovering a new particle with rates near that of the SM Higgs. We consider models with a signal in $\gamma \gamma$ below the SM Higgs mass. We discuss singlet models with additional vectorlike matter, but argue that a Type-I two Higgs doublet model can more easily provide detectable rates. In such scenarios, in regions of moderate-to-strong fermiophobia, the enhanced $\gamma \gamma$ branching ratio allows signals from $V H+\mathrm{VBF}$ production to yield $\sigma \times B R_{\gamma \gamma}$ comparable to total SM rates and would thus be detectable. Light $H$ production can be dominated via rare top decays $t \rightarrow b H^{+} \rightarrow b W^{*} H$, which provides an even more efficient means of production. We also consider this in the context of various Higgs anomalies, specifically the recent $2.9 \sigma$ (local) CMS excess at $95 \mathrm{GeV}$, the LEP Higgs excess near the same mass, and excesses in $t \bar{t} h$ searches at Tevatron and LHC. We find regions of parameter space that can meet all simultaneously. An implication of the Type-I scenario is that any $\gamma \gamma$ excess should be associated with additional elements that could reduce background, including $b$-jets, forward jets or signs of vector boson production.

Keywords: Beyond Standard Model, Higgs Physics

ArXiv EPrint: 1710.07649 


\section{Contents}

1 Introduction 1

2 Signals of light from a lighter Higgs $\quad 2$

2.1 Higgs signals from singlets 2

2.2 Type-I two Higgs Doublet models 3

$\begin{array}{lll}3 & \text { Global perspective of other anomalies } & 7\end{array}$

3.1 Explaining the excesses with a Type-I 2HDM 8

4 Discussion $\quad 10$

\section{Introduction}

The search for the Higgs boson was a tremendous undertaking. Not just at the LHC, but in the decades and experiments that preceded it. Results from LEP and the Tevatron provided the basis on which the multi-channel searches at the LHC proceeded. With the Higgs now discovered, and the LHC awaiting more luminosity — but little more energy it is worth turning some attention to understanding what sorts of particles might still lie hidden in these data.

The simplest reason to pursue this is straightforward - at the LHC, one expects a massive increase in luminosity, and thus sensitivity, to new states, even with couplings well below $\mathcal{O}(1)$. With no dramatic increases in energy on the horizon, our best hope for discoveries is in the ever-improving statistics that comes with these new data. At the same time, new ideas of what may lie there can point us to new signals that can help improve sensitivity of ongoing searches.

Indeed, throughout the search for the Higgs, there have been a variety of tantalizing bumps and excesses, many of which have lingered as open questions on the myriad exclusion plots presented over the years. If any of these point to physics beyond the standard model, statistics alone may not be enough for discovery.

Amongst these bumps comes the most recent result from CMS [1], which shows a small excess near $95 \mathrm{GeV}$ in the diphoton channel. We begin our discussion, in section 2, describing models that can yield rates that are discoverable in the near term at the LHC. In particular, we use the CMS excess as a guide to characterize sensitivity. Many of the scenarios predict diphoton rates near experimental sensitivity, but also predict associated elements that have not been previously discussed and can dramatically boost sensitivity of LHC searches to, specifically, Type I 2HDM singlets. In section 3 how some of these models may also explain historical excesses from LEP and the Tevatron, as well as excesses in other channels at the LHC. 
In section 4 we conclude, and emphasize how, irrespective of any anomaly, these models provide insights that can direct improvements to existing searches at the LHC. In particular, by adding tags for forward jets, vector bosons or associated b-jets to the diphoton signal, dramatic improvements of sensitivity can be achieved.

\section{Signals of light from a lighter Higgs}

Recently, the CMS collaboration has reported a significant improvement in its search for the diphoton resonances in the light mass regime $(70-110 \mathrm{GeV})[1]$. With $35.9 \mathrm{fb}^{-1}$ at $13 \mathrm{TeV}$, they have remarkably passed the critical threshold of being sensitive to models with SM-strength in this mass regime, opening up sensitivity to new models. In this analysis, CMS has reported a $2.9 \sigma$ (local) excess at $95.3 \mathrm{GeV}$. The overall rate is consistent with a production cross section $\sigma_{p p \rightarrow H} B R_{H \rightarrow \gamma \gamma} \simeq 0.1 \mathrm{pb}$, which is similar to the SM rate expected at that mass. This excess has already drawn attention $[2,3]$. Of course, we know from LEP that no SM-like Higgs boson exists at that mass [4]. It is worth considering what kinds of models could achieve this, and whether they predict any new features that could improve the searches further. Thus, using this as a concrete target, one can ask what sorts of models can create a diphoton resonance with a cross section approaching that of the SM Higgs boson at the LHC. ${ }^{1}$

\subsection{Higgs signals from singlets}

A simple example is that of a singlet scalar, $\phi$, which has been extensively discussed (see e.g., [5-12]). To allow for production from gluon fusion at the LHC the singlet must couple to extra vectorlike colored matter, $y \phi \bar{\Psi} \Psi$, necessitating the introduction of many new degrees of freedom. If that matter is also electrically charged, then the decay to photons is automatic. Such particles that can appear at near-SM rates — but are easily distinguished from a SM Higgs - have been referred to previously as Higgs friends [12]. In another context, in a higher mass regime around $700-800 \mathrm{GeV}$, this has been referred to as the "everybody's model" [13].

The production cross section for such a particle can be related to its gluon decay width, and these quantities for a SM Higgs of the same mass,

$$
\sigma_{\phi}=\sigma_{h} \times \frac{\Gamma_{\phi \rightarrow g g}}{\Gamma_{h \rightarrow g g}}
$$

where

$$
\Gamma_{\phi \rightarrow g g}=\frac{y^{2} \alpha_{s}^{2} N_{\Psi}^{2} m_{\phi}^{3}}{72 \pi^{3} m_{\Psi}^{2}} .
$$

Where we assume $N_{\Psi}$ copies of a vectorlike Dirac color triplet fermion, with mass $m_{\Psi}$.

\footnotetext{
${ }^{1} \mathrm{It}$ is worth noting that the $8 \mathrm{TeV}$ data sees a smaller excess at a different mass. Because of the strong energy dependence of the cross sections in the models we will consider, the expected cross section at $8 \mathrm{TeV}$ would be $2-3$ times lower than that at $13 \mathrm{TeV}$. A marginal detection at $13 \mathrm{TeV}$ would not have produced a sizable signal at $8 \mathrm{TeV}$, even in the SM. In the models we consider, where the energy dependence is often stronger, it would be misleading to naively use the $8 \mathrm{TeV}$ results or combine them with $13 \mathrm{TeV}$.
} 
The overall cross section times branching ratio is then:

$$
\sigma(g g \rightarrow \phi \rightarrow \gamma \gamma)=\sigma_{h} \times \frac{\Gamma_{\phi \rightarrow \gamma \gamma}}{\Gamma_{h \rightarrow g g}}
$$

where

$$
\Gamma_{\phi \rightarrow \gamma \gamma}=\frac{y^{2} \alpha^{2} N_{\Psi}^{2} Q_{\Psi}^{4} m_{\phi}^{3}}{16 \pi^{3} m_{\Psi}^{2}}=y^{2} N_{\Psi}^{2}\left(\frac{Q_{\Psi}}{\frac{2}{3}}\right)^{4}\left(\frac{m_{\phi}}{95 \mathrm{GeV}}\right)^{3}\left(\frac{200 \mathrm{GeV}}{m_{\Psi}}\right)^{2} \times 0.5 \mathrm{keV}
$$

for $N_{\Psi}$ fermions with charge $Q_{\Psi}$. A $95 \mathrm{GeV}$ SM-like Higgs boson has a gluon fusion production cross section of $76.3 \mathrm{pb}$ and a width into gluons of $0.15 \mathrm{MeV}$. Thus,

$$
\sigma_{\phi} B R_{\phi \rightarrow \gamma \gamma} \approx 0.5 \mathrm{pb} \times y^{2} N_{\Psi}^{2}\left(\frac{Q_{\Psi}}{\frac{2}{3}}\right)^{4}\left(\frac{200 \mathrm{GeV}}{m_{\Psi}}\right)^{2} .
$$

Thus, a signal of the size seen at CMS is still possible, but it requires new light colored particles. Even a new colored fermion as light as $200 \mathrm{GeV}$ could have escaped detection so far at the LHC, if it decays predominantly into three jets [14]. However, while it appears one can evade LHC bounds on colored particles and still have a sizable signal, it is certainly not economical to add new states both to observe and explain the production. Moreover, although we have not yet discussed them, such a model cannot hope to easily explain the other Higgs related anomalies present in the data.

An alternative approach to adding a singlet and new colored fermions is instead to mix the singlet, $s$, with the Higgs boson. In such a case, the light mass eigenstate's couplings to SM fields will be proportional to some mixing angle $\sin \delta$ (hereafter $s_{\delta}$ ). The dominant production of $s$ will be through gluon fusion, but will occur at a rate suppressed by $s_{\delta}^{2}$. Furthermore, as the dominant branching ratio $(s \rightarrow b \bar{b})$ is also proportional to the fermion coupling, the rate to $\gamma \gamma$ is independent of this mixing,

$$
\sigma(p p \rightarrow s \rightarrow \gamma \gamma) \simeq s_{\delta}^{2} \sigma_{p p \rightarrow h} \times \frac{\Gamma_{s \rightarrow \gamma \gamma}}{s_{\delta}^{2} \Gamma_{h \rightarrow b b}} .
$$

Thus, the rate to produce $s$ in the diphoton channel is directly proportional to the $\gamma \gamma$ width of $s$. Achieving a rate comparable to the SM Higgs then requires $s$ having a diphoton width comparable to the SM Higgs (i.e. $\Gamma(\gamma \gamma) \approx 0.5 \mathrm{keV}$ ), which is not possible through mixing alone. As we can see from (2.4), this is possible, if $s$ has $\mathcal{O}(1)$ couplings to additional light fermions which have $\mathcal{O}(1)$ electric charge.

\subsection{Type-I two Higgs Doublet models}

Perhaps the most economical model that can produce a detectable diphoton resonance is the Type-I two Higgs Doublet model (see discussion in [15]). This model consists of two $\mathrm{SU}(2)$ scalar doublets, $\Phi_{1,2}$ which have opposite charge under a discrete $\mathbb{Z}_{2}$ symmetry, we take both to have hypercharge $Y=1 / 2$. All right-handed SM fermions are even under the $\mathbb{Z}_{2}$ which means that one doublet, $\Phi_{1}$, is fermiophobic. Such a model provides some additional freedom in its couplings to gauge bosons and fermions, and contains already a charged scalar which can mediate new processes. 
We parametrize the two doublets as

$$
\begin{gathered}
\Phi_{1}=\left(\begin{array}{c}
-H^{+} s_{\beta}+G^{+} c_{\beta} \\
\frac{1}{\sqrt{2}}\left(v c_{\beta}-h s_{\alpha}+H c_{\alpha}-i A^{0} s_{\beta}+i G^{0} c_{\beta}\right)
\end{array}\right), \\
\Phi_{2}=\left(\begin{array}{c}
H^{+} c_{\beta}+G^{+} s_{\beta} \\
\frac{1}{\sqrt{2}}\left(v s_{\beta}+h c_{\alpha}+H s_{\alpha}+i A^{0} c_{\beta}+i G^{0} s_{\beta}\right)
\end{array}\right) .
\end{gathered}
$$

With $h$ corresponding to the Higgs observed at $125 \mathrm{GeV}$, and $H$ its CP-even partner. The tree-level couplings of the Higgs mass eigenstates to fermions, relative to the coupling of a SM Higgs are

$$
c_{f}^{h}=\frac{c_{\alpha}}{s_{\beta}}=c_{\delta}-\frac{s_{\delta}}{t_{\beta}}, \quad c_{f}^{H}=\frac{s_{\alpha}}{s_{\beta}}=-\left(s_{\delta}+\frac{c_{\delta}}{t_{\beta}}\right), \quad c_{u}^{A^{0}}=-c_{d, \ell}^{A^{0}}=\frac{1}{t_{\beta}},
$$

Where we have introduced the angle $\delta=\beta-\alpha-\pi / 2$ to parametrize the deviation of the Higgs couplings from SM values [16]. Similarly the couplings to gauge bosons are

$$
c_{V}^{h}=s_{\beta-\alpha}=c_{\delta}, \quad c_{V}^{H}=c_{\beta-\alpha}=-s_{\delta} .
$$

The cross sections and widths of the new Higgs boson vary differently depending on these angles. Normalizing to a $m_{H}=95 \mathrm{GeV}$, there are first those that scale with $\left(s_{\alpha} / s_{\beta}\right)^{2}$,

$$
\begin{aligned}
\sigma(g g) & =76.3 \mathrm{pb} \times\left(\frac{s_{\alpha}}{s_{\beta}}\right)^{2}, & \sigma(t \bar{t} H)=1 \mathrm{pb} \times\left(\frac{s_{\alpha}}{s_{\beta}}\right)^{2}, \\
\sigma(b \bar{b} H) & =1 \mathrm{pb} \times\left(\frac{s_{\alpha}}{s_{\beta}}\right)^{2}, & \\
\Gamma(b \bar{b}) & =1.9 \mathrm{MeV} \times\left(\frac{s_{\alpha}}{s_{\beta}}\right)^{2}, & \Gamma\left(\tau^{+} \tau^{-}\right)=0.2 \mathrm{MeV} \times\left(\frac{s_{\alpha}}{s_{\beta}}\right)^{2}, \\
\Gamma(g g) & =0.15 \mathrm{MeV} \times\left(\frac{s_{\alpha}}{s_{\beta}}\right)^{2}, &
\end{aligned}
$$

and also those that are proportional to $s_{\delta}^{2}$,

$$
\begin{array}{llrl}
\sigma(V B F)=5 \mathrm{pb} \times s_{\delta}^{2}, & \sigma(W H)=3.4 \mathrm{pb} \times s_{\delta}^{2}, & \sigma(Z H)=2 \mathrm{pb} \times s_{\delta}^{2}, \\
\Gamma\left(W W^{*}\right)=0.01 \mathrm{MeV} \times s_{\delta}^{2}, & \Gamma\left(Z Z^{*}\right)=1.6 \mathrm{MeV} \times 10^{-3} s_{\delta}^{2} .
\end{array}
$$

Finally, the diphoton partial width is

$$
\Gamma(\gamma \gamma)=\left|1.31 s_{\delta}+0.31 \frac{s_{\alpha}}{s_{\beta}}\right|^{2} \times 3.27 \times 10^{-3} \mathrm{MeV}
$$

Other interesting channels are $H H^{+}$production through an off-shell $W$ boson [17-21] and $Z H$ production through an off-shell $A^{0}$ [22]. The couplings of the $125 \mathrm{GeV}$ Higgs boson are constrained to lie close to SM values [23] which means that $\left|s_{\delta}\right| \lesssim 0.4$. We will consider regions of mild fermiophobia, where the coupling of $H$ to fermions is suppressed. 


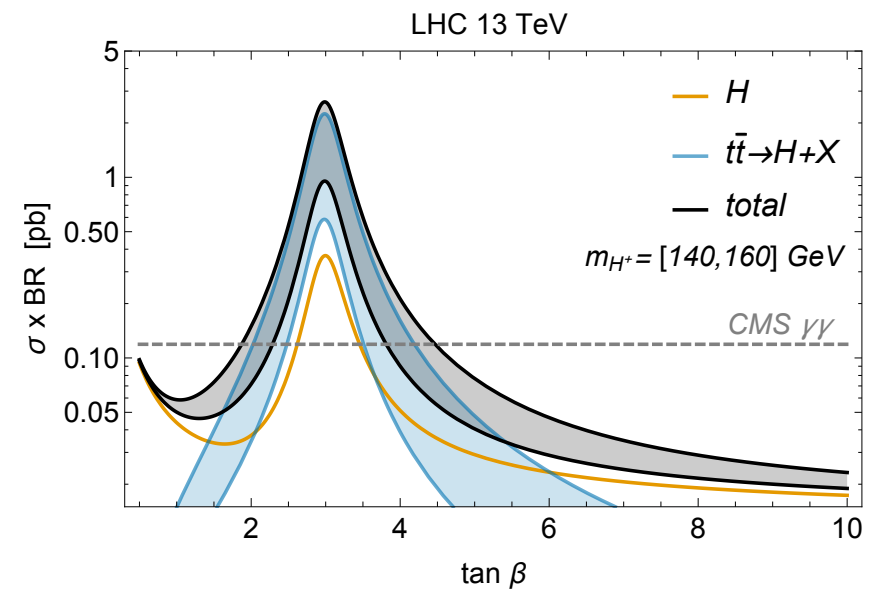

Figure 1. Production rate, $\sigma_{p p \rightarrow H} \times \mathrm{BR}_{\mathrm{H} \rightarrow \gamma \gamma}$, of a $95 \mathrm{GeV}$ Higgs of a Type-1 $2 \mathrm{HDM}$ at $13 \mathrm{TeV}$ LHC assuming $s_{\delta}^{2}=0.1$. The lower (brown) curve is from all mixing induced processes (ggF, VBF, $\mathrm{VH}$ ), the lower (blue) band is the contribution from rare cascade decays of tops with the charged Higgs mass in the range $[140,160] \mathrm{GeV}$, and the upper (black) band is the sum of both contributions. The dashed line shows the rate needed to explain the CMS excess at $95 \mathrm{GeV}$.

Thus, for the remainder of this paper we will consider the region $s_{\delta}<0$, the region $s_{\delta}>0$ is the region of (mild) fermiophilia. We define the ratio $f_{\mathrm{FP}}^{2}=s_{\delta}^{2} /\left(s_{\alpha}^{2} / s_{\beta}^{2}\right)$ as the "factor of fermiophobia" and consider fermiophobic regions to be those where $f_{\mathrm{FP}}^{2} \gg 1$. In these regions, the branching ratio to diphoton is enhanced

$$
B R_{H \rightarrow \gamma \gamma} \simeq f_{\mathrm{FP}}^{2}\left(I_{W W}\left(m_{H}\right)+\frac{1}{f_{\mathrm{FP}}} I_{t t}\left(m_{H}\right)\right)^{2},
$$

where $I_{W W}\left(m_{H}\right)$ and $I_{t t}\left(m_{H}\right)$ are mass dependent functions resulting from loop integrals. For a $95 \mathrm{GeV}$ Higgs boson, $I_{W W}(95 \mathrm{GeV}) \approx 0.05$ and $I_{t t}(95 \mathrm{GeV}) \approx 0.01$, and in the fermiophobic regime the branching ratio to diphoton is $B R_{H \rightarrow \gamma \gamma} \simeq 3 f_{\mathrm{FP}}^{2} \times 10^{-3}$. The approximate relationship above assumes the $b \bar{b}$ decay still dominates the total width.

Using the results above, we see that, in the fermiophobic limit, the rate for $H \rightarrow \gamma \gamma$ through gluon fusion production scales as

$$
\sigma_{p p \rightarrow H \rightarrow \gamma \gamma}^{g g} \approx 0.2 s_{\delta}^{2} \mathrm{pb} .
$$

With various bounds limiting $s_{\delta}^{2} \lesssim 0.1$, this is a small fraction of the needed rate. In contrast, $\mathrm{VBF} / \mathrm{VH}$ production processes scale as

$$
\sigma_{p p \rightarrow H \rightarrow \gamma \gamma}^{(\mathrm{VBF} / \mathrm{VH})}=0.03 s_{\delta}^{2} f_{\mathrm{FP}}^{2} \mathrm{pb} .
$$

For $f_{\mathrm{FP}}^{2} \gtrsim 7$, the fermiophobic regime, gluon fusion will no longer be the dominant production channel for $H \rightarrow \gamma \gamma$. For strong fermiophobia $f_{f p}^{2} \sim 20-40$, total cross sections of $\mathcal{O}(0.1 \mathrm{pb})$ are possible, thus explaining the CMS excess. We show the directly produced signal in figure 1 , and see that it can be large enough to explain the CMS excess for values of $\tan \beta$ around 3 . 

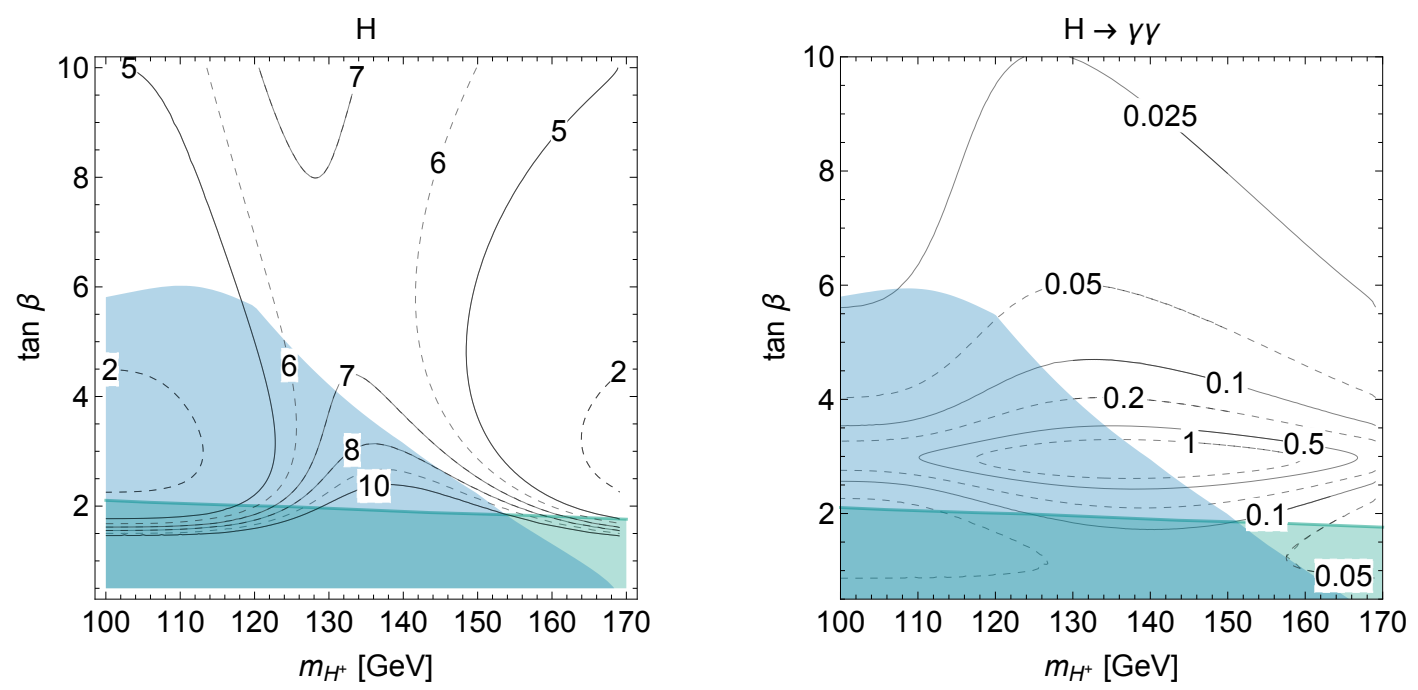

Figure 2. Contours of $H$ production cross section, $\sigma_{H}$, in pb (through direct $H$ production as well as production through top decay) at $13 \mathrm{TeV}$ LHC, as a function of charged Higgs mass, $m_{H^{+}}$ and $\tan \beta$ (left); production cross section times branching ratio to $\gamma \gamma, \sigma_{H} B R_{H \rightarrow \gamma \gamma}$ (right). In both figures the large (blue) region is ruled out by searches for rare top decays $t \rightarrow b\left(H^{+} \rightarrow \tau^{+} \nu\right)$ and the smaller (green) region is ruled out by flavor observables $\left(\Delta M_{s}\right.$ and $\left.B_{s}^{0} \rightarrow \mu^{+} \mu^{-}\right)$. We have taken the $A^{0}$ to be heavier than the top quark and $s_{\delta}^{2}=0.1$.

This discussion has so far focused on tree-level changes to the Higgs branching ratio to photons. However, the light Higgs also couples to the charged Higgs. One expects a loop of charged Higgses and a resulting contribution to the width

$$
\delta \Gamma_{H \rightarrow \gamma \gamma}=\frac{\alpha^{2} m_{H}^{3}}{2304 \pi^{3} m_{H^{+}}^{2}}\left|\frac{d m_{H^{+}}}{d\langle H\rangle}\right|^{2} \approx\left|\frac{d m_{H^{+}}}{d\langle H\rangle}\right|^{2}\left(\frac{m_{H}}{95 \mathrm{GeV}}\right)^{3}\left(\frac{140 \mathrm{GeV}}{m_{H^{+}}}\right)^{2} 40 \mathrm{eV} .
$$

Where by $\frac{d m_{H^{+}}}{d\langle H\rangle}$ we mean the derivate of the (field dependent) charged Higgs mass with respect to the piece of the vev that aligns with the mass eigenstate $H$. In the fermiophobic limit with $s_{\delta}^{2}=0.1$, the width from top and $\mathrm{W}$ boson loops gives $\Gamma_{H \rightarrow \gamma \gamma} \simeq 0.6 \mathrm{keV}$. Thus, for $\left|\frac{d m_{H^{+}}}{d\langle H\rangle}\right| \lesssim \mathcal{O}(1)$, the charged-Higgs loop is smaller than the SM contributions. The exact size of this contribution is, however, very model dependent, and depends upon which operators split the charged Higgs from the neutral ones. Typically, $\left|\frac{d m_{H^{+}}}{d\langle H\rangle}\right|<1$ and the loop corrections from $\mathrm{H}^{+}$are not large.

There is another production possibility, again involving a light charged Higgs, that was recently emphasized by [24]. Namely, that the light scalar production can occur in cascades from a heavier charged Higgs [25-30]. In a Type-I model, in the presence of a charged Higgs below the top mass, $m_{H^{+}}<m_{t}$, [24] showed that the production of the light Higgs via $t \rightarrow H^{+} b \rightarrow H W^{*} b$, could be sizable, and consistent with existing constraints. For moderate $\tan \beta \lesssim 6$, the branching ratio for this process can be $B R\left(t \rightarrow H W^{*} b\right) \sim \mathcal{O}(0.01)$, despite the decay of $H^{+}$being three body. The top production cross section at $13 \mathrm{TeV}$ is $\sim 830 \mathrm{pb}$, which offers a $H$ production cross section of $\mathcal{O}(10 \mathrm{pb})$. This can yield a CMS signal in the mildly fermiophobic regime (i.e., $f_{\mathrm{FP}}^{2} \sim 5$ ), with $B R_{H \rightarrow \gamma \gamma} \sim 10^{-2}$. We show 
this combined signal in figure 1 , and again there is a region at moderate $\tan \beta$ where the produces rates comparable to a SM Higgs at the same mass.

We stress, if this channel $p p \rightarrow t \bar{t}, t \rightarrow H^{+} b, H^{+} \rightarrow H^{0} W^{*}, H^{0} \rightarrow \gamma \gamma$ is available it will dominate production beyond previously considered sources of associated with VBF and one would expect additional signals in the LHC events. Specifically, with two associated b-jets present in the event, even a weak b-tag would provide an efficient means to lower background significantly and provide new constraints on this regime of Type I 2HDMs.

There are several constraints on new light Higgs bosons that limit the available parameter space. These constraints are weaker for a Type-I 2HDM than for Type-II. Due to mass splittings among components of the Higgs doublets there are contributions to the precision electroweak observables $S$ and $T$, however these constraints are weak. There are indirect constraints from $B$-physics observables e.g. $\Delta M_{s}, B_{s}^{0} \rightarrow \mu^{+} \mu^{-}, b \rightarrow$ s $\gamma$, etc [31]. The strongest constraint over most of the parameter space we are interested in comes from searches for $t \rightarrow b\left(H^{+} \rightarrow \tau^{+} \nu\right)$ [32]. We note that while one might have expected stronger constraints from $\mathrm{H}^{+} \rightarrow \tau^{+} \nu$, the channel $\mathrm{H}^{+} \rightarrow W^{*} H^{0} / A^{0}$ often dominates, weakening those limits [24].

\section{Global perspective of other anomalies}

With so many Higgs searches, it is perhaps not surprising that a number of anomalies have arisen. Here we provide a brief discussion of a few of them and how one might attempt to explain them simultaneously.

LEP anomaly. Using approximately $2.5 \mathrm{fb}^{-1}$ of data taken across a range of energies, $189 \mathrm{GeV}<\sqrt{s}<209 \mathrm{GeV}$, the four LEP experiments searched for the process $e^{+} e^{-} \rightarrow Z H$ where the Higgs boson decays into $b$ jets or tau leptons. Combining all data [4], the experiments saw a broad excess $(>2 \sigma)$ above background expectations between $95 \mathrm{GeV}$ and $100 \mathrm{GeV}$, with the largest deviation at $m_{H}=99 \mathrm{GeV}$. LEP was most sensitive to $H \rightarrow b \bar{b}$ and this excess corresponds to a rate to $Z b \bar{b}$ of $\sim 0.1$ of the SM rate for a Higgs in the same mass range, i.e. $\xi^{2} \equiv\left(g_{H Z Z} / g_{H Z Z}^{\mathrm{SM}}\right)^{2} \approx 0.1$.

LHC $\gamma \boldsymbol{\gamma}$. The CMS collaboration has carried out a search for diphoton resonances in the range $[80,110] \mathrm{GeV}$ using $35.9(19.7) \mathrm{fb}^{-1}$ of $\sqrt{s}=13$ (8) $\mathrm{TeV}$ data $[1,33]$. The combination of the two data sets has its largest discrepancy from SM background at $m_{H}=95.3 \mathrm{GeV}$, corresponding to a local (global) significance of $2.8 \sigma(1.3 \sigma)$. At this mass the $95 \%$ confidence limit on the Higgs production cross section times branching ratio at $\sqrt{s}=13(8) \mathrm{TeV}$ is approximately $0.1(0.05) \mathrm{pb}$. At present ATLAS only has a search for diphoton resonances with $m_{\gamma \gamma}<110 \mathrm{GeV}$ for $20.3 \mathrm{fb}^{-1}$ at $\sqrt{s}=8 \mathrm{TeV}$ [34], and no public analysis using $\sqrt{s}=13 \mathrm{TeV}$ data. From this analysis the $95 \%$ confidence limit on the Higgs production cross section times branching ratio at $m_{\gamma \gamma} \approx 95 \mathrm{GeV}$ is $\sigma B R \lesssim 0.05 \mathrm{pb}$.

LHC and Tevatron $\boldsymbol{t} \bar{t} \boldsymbol{H}$. A variety of searches have been performed for a Higgs boson produced in association with a top quark pair. Notably, some of these have seen excesses [35, 36], while others have not [37-40]. Generally, more inclusive analyses (those employing cut-and-count approaches and sensitive to the specific value of $m_{h}=125 \mathrm{GeV}$ ) have seen greater excesses, while more exclusive analyses (those employing highly trained 
BDTs or neural nets or demanding $m_{h}=125 \mathrm{GeV}$ ) have not. We refer the reader to [24] for a thorough discussion.

A few important points are relevant, however: a search for $t \bar{t} H$ was performed by $\mathrm{CDF}[41,42]$ with $t \bar{t} H \rightarrow W W b \bar{b} b \bar{b}$ in the range $100 \mathrm{GeV}<m_{h}<150 \mathrm{GeV}$. Because of the combinatorics of $b$ jets, the ability to discriminate a Higgs mass peak was poor. Nonetheless, the search shows a weak excess, reaching $\mathcal{O}(2 \sigma)$ near $100 \mathrm{GeV}$. At the LHC, ATLAS saw an excess in their multilepton analysis, which was cut and count, while CMS, employing a BDT did not.

Critically, both experiments truncated their most focused $t \bar{t} H$ and VBF $h \rightarrow \gamma \gamma$ analyses at a point that a $95 \mathrm{GeV}$ boson would have been missed (CMS has a lower bound of $100 \mathrm{GeV}$ while ATLAS goes down to $105 \mathrm{GeV}$ ). The CMS low mass search [1] was sensitive to $t \bar{t} H, V h$, and VBF production mechanisms in addition to gluon fusion, but did not break out separate analyses for them, setting limits based on their expected relative rate and efficiencies in the SM.

\subsection{Explaining the excesses with a Type-I 2HDM}

It is clear that one can explain any one of the excesses, for instance with a new singlet coupled to vectorlike fermions, but an intriguing question is whether one can explain most or even all of the excesses in a compact model. As discussed in section 2.2 it is possible to explain the CMS $\gamma \gamma$ bump at $95 \mathrm{GeV}$ in a Type-I $2 \mathrm{HDM}$ in the region of fermiophobia. We shall argue that such a Type-I 2HDM provides a simple explanation for all excesses, while being consistent with null results.

The LEP results [4] are most simply understood as a type of scalar mixing with the Higgs boson at a level $s_{\delta}^{2} \sim 0.1$. However, this could be an $\mathrm{SU}(2)$ singlet or doublet scalar field. Producing a $\gamma \gamma$ signal at the LHC comparable to the SM with such a small mixing is a challenge, however. Absent new colored particles, one must boost the production cross section via mixing with the SM Higgs. Since such rates are necessarily below the SM, we must in turn resort to enhancing the $\gamma \gamma$ width of the new state.

As shown earlier (2.14), going to the fermiophobic regime, $f_{\mathrm{FP}} \gg 1$, increases $B R_{H \rightarrow \gamma \gamma}$. With the requirement from LEP that $s_{\delta}^{2} \sim 0.1$, we must go into the strongly fermiophobic regime, where $f_{f p}^{2} \approx 20-40$. Then we find a signal at the LHC of $\sigma_{\mathrm{HV}+\mathrm{VBF}} \times B R_{H \rightarrow \gamma \gamma} \sim$ $0.1 \mathrm{pb}$, while the rate from gluon fusion is considerably smaller, see (2.15) and (2.16). That is, the CMS $\gamma \gamma$ excess can be explained not by $g g F$ but instead by the combination of VBF and associated production [43], which all lead to events with additional activity and other signals. We show the consistent region of parameter space in figure 3 (left).

If the charged Higgs present in 2HDM's is lighter than the top mass, there is an even more exciting possibility. This scenario, first discussed in [24], has the dominant light Higgs production via $t \rightarrow H^{+} b \rightarrow H W^{*} b$. It was argued in [24] that this process, involving a final state very similar to $t \bar{t} h$, would be a natural contaminant of those searches, and, indeed, could provide the explanation of the excesses seen. For a charged Higgs mass in the range $140 \mathrm{GeV}<m_{H^{+}}<160 \mathrm{GeV}$, one needs $\tan \beta \approx 5$ to explain the $t \bar{t} h$ signals. Unfortunately, to explain the LEP excess in this $\tan \beta$ regime, one is naturally in the moderately fermiophobic regime, and is a non-trivial consistency check of this scenario. While 

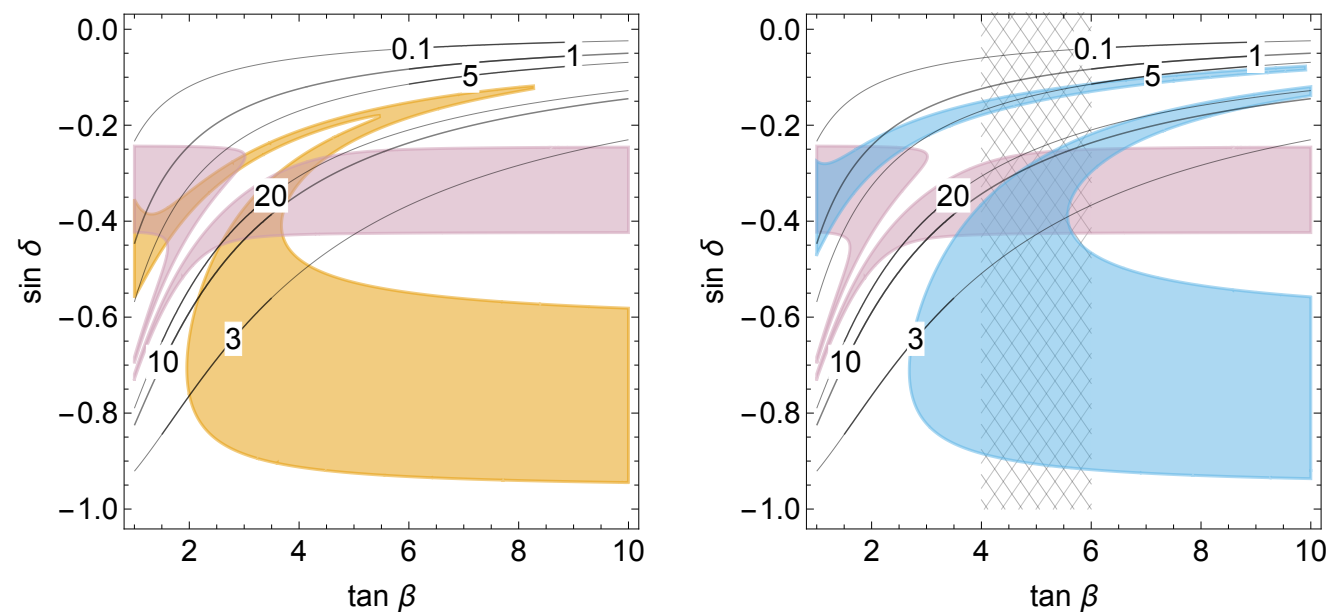

Figure 3. Contours of $f_{\mathrm{FP}}^{2}$ as a function of $s_{\delta}$ and $\tan \beta$. Pink regions show areas consistent with the LEP excess $\left(0.05<\xi^{2}<0.15\right)$ while the brown (left) or blue (right) region shows the area consistent with the CMS excess $\left(0.05 \mathrm{pb}<\sigma B R_{H \rightarrow \gamma \gamma}<0.1 \mathrm{pb}\right)$. Left $-H$ production arising only from $\mathrm{ggF}, \mathrm{VBF}, \mathrm{VH}$ processes. Right $-H$ production including cascade decays from top quarks with $m_{H^{+}}=140 \mathrm{GeV}$. For the right plot, the approximate range (hashed) to explain the leptonic $t \bar{t} h$ excesses is $4 \lesssim \tan \beta \lesssim 6[24]$. For $s_{\delta}>0$, the Higgs is fermiophilic (i.e., $f_{\mathrm{FP}}^{2}<1$ ) and the $\gamma \gamma$ rates are suppressed.

it was noted by [24] that one could explain the LEP and $t \bar{t} h$ signals simultaneously, the near-inevitable boosted $\gamma \gamma$ signal was not recognized at the time. The global consistency of all three anomalies is shown in figure 3 (right), for $m_{H^{+}}=140 \mathrm{GeV}$. Note that increasing the charged Higgs mass shifts the required region for both CMS $\gamma \gamma$ and $t \bar{t} h$ to smaller $\tan \beta$. This is compatible with the constraints on $\tan \beta$ coming from rare top decays and indirect constraints from $B$ physics, see figure 2. The same figure shows that to explain the anomalies there is a lower bound on the charged Higgs mass $m_{H^{+}} \gtrsim 130 \mathrm{GeV}$ and an upper bound on the $H$ production cross section $\sigma_{H} \lesssim 10 \mathrm{pb}$.

If the top decay to $H^{+}$is open then production of $H$ through top decay dominates over the sum of ggF, VBF, and VH meaning that there should be considerable additional activity in the excess $\gamma \gamma$ events e.g. $b$ jets and leptons. Furthermore, in the $t \bar{t} h$ searches there should also be a $\gamma \gamma$ resonance at $95 \mathrm{GeV}$. Remarkably, the CMS and ATLAS searches for $\mathrm{VBF}$ and $t \bar{t} h$ with $h \rightarrow \gamma \gamma$ stopped short of going into this mass range.

Because the $B R(H \rightarrow \gamma \gamma)$ is so much larger than in the SM, the expected rate is an order of magnitude - or more - beyond what is expected from the SM. Assuming the efficiency to pass the analysis cuts for a $95 \mathrm{GeV}$ Higgs is comparable to the SM one expects a considerable number of signal events just below the existing analysis range. The resolution of the diphoton invariant mass is $\sim 1.5 \mathrm{GeV}$ so a small fraction of the events centered around $95 \mathrm{GeV}$ will leak into the analysis window, but this is too small to have been observed. It almost defies belief, but the natural implication of this scenario is that there is an enormous signal lying just outside the currently searched mass window. While this seems unlikely, we cannot find any published paper or note that precludes this exciting possibility. 


\section{Discussion}

With the increasing sensitivity of Higgs searches, we confront the prospect of the discovery of new particles with Higgs-like properties. Simple models can provide signals into diphotons at rates comparable to the SM. Singlets can still provide high rates, but need additional light fields to provide production and/or widths to $\gamma \gamma$. In contrast, a doublet mixing with the SM Higgs in the form of a Type-I 2HDM provides an economical model that provides a boosted $\gamma \gamma$ signal in the fermiophobic regime of parameters. In the simplest case, the signal is generated not by $g g F$ but by $\mathrm{VBF}+\mathrm{VH}$ production, and thus would offer additional tags to improve separation of signal and background.

This last possibility is particularly exciting when viewed through the lens of a series of anomalies in Higgs searches, which could all be arise from such a model. In particular, for a Type I $2 \mathrm{HDM}$ in the light mass regime, we point out that $\gamma \gamma$ could provide the strongest sensitivity. When kinematically available, the neutral scalar production can arise in the manner proposed by [24], where the light Higgs is produced in a cascade $t \rightarrow H^{+} b \rightarrow b W^{+*} H$. Leptonic decays of the $H^{0}$ naturally contaminate the $t \bar{t} H$ searches, but in much of the parameter space the light Higgs is somewhat to very fermiophobic, and the $\gamma \gamma$ rate is enhanced. In such case, lowering the mass threshold for $t \bar{t} H, H \rightarrow \gamma \gamma$ searches, or looking for additional tags in conventional $H \rightarrow \gamma \gamma$ searches should yield dramatic signals well above SM rates and would provide the best test of this region of Type I 2HDM.

Indeed, even absent any anomalies, the analysis presented here suggests that dramatically improved sensitivity to Type I $2 \mathrm{HDM}$ could be achieved by any of the following: lowering the $H$ mass threshold for $t \bar{t} H$ or VBF $H$ production with $H \rightarrow \gamma \gamma$, or by adding additional b-jet tags to light $H \rightarrow \gamma \gamma$ searches.

In summary, it is clear the prospect for discovery of new states in Higgs searches is significant. Simple modifications or extensions of existing searches can improve their sensitivities to Type I $2 \mathrm{HDM}$ in the light mass regime. Moreover, if any of the anomalies above survive after further scrutiny and data, it may be that Higgs searches are not only the searches that completed the Standard Model, but may be the ones that find the first physics beyond it, as well.

\section{Acknowledgments}

We thank Anna Maria Taki for helpful discussions. The work of NW is supported by the Simons Foundation and by the NSF under grant PHY-1620727. This work was supported by the DoE under contract number DE-SC0007859 and Fermilab, operated by Fermi Research Alliance, LLC under contract number DE-AC02-07CH11359 with the United States Department of Energy. PJF would like to thank the CCPP and NYU for kind hospitality while this work was initiated. The Flatiron Institute is supported by the Simons Foundation. 
Open Access. This article is distributed under the terms of the Creative Commons Attribution License (CC-BY 4.0), which permits any use, distribution and reproduction in any medium, provided the original author(s) and source are credited.

\section{References}

[1] CMS collaboration, Search for new resonances in the diphoton final state in the mass range between 70 and $110 \mathrm{GeV}$ in pp collisions at $\sqrt{s}=8$ and $13 \mathrm{TeV}$, CMS-PAS-HIG-17-013 (2017).

[2] A. Mariotti, D. Redigolo, F. Sala and K. Tobioka, New LHC bound on low-mass diphoton resonances, arXiv: 1710.01743 [INSPIRE].

[3] A. Crivellin, J. Heeck and D. Müller, Large $h \rightarrow$ bs in generic two-Higgs-doublet models, Phys. Rev. D 97 (2018) 035008 [arXiv:1710.04663] [INSPIRE].

[4] OPAL, DelPhi, LEP Working Group for Higgs boson searches, ALEPH, L3 collaboration, R. Barate et al., Search for the standard model Higgs boson at LEP, Phys. Lett. B 565 (2003) 61 [hep-ex/0306033] [INSPIRE].

[5] W.D. Goldberger and M.B. Wise, Phenomenology of a stabilized modulus, Phys. Lett. B 475 (2000) 275 [hep-ph/9911457] [INSPIRE].

[6] G.F. Giudice, R. Rattazzi and J.D. Wells, Graviscalars from higher dimensional metrics and curvature Higgs mixing, Nucl. Phys. B 595 (2001) 250 [hep-ph/0002178] [INSPIRE].

[7] C. Csáki, M.L. Graesser and G.D. Kribs, Radion dynamics and electroweak physics, Phys. Rev. D 63 (2001) 065002 [hep-th/0008151] [INSPIRE].

[8] W.D. Goldberger, B. Grinstein and W. Skiba, Distinguishing the Higgs boson from the dilaton at the Large Hadron Collider, Phys. Rev. Lett. 100 (2008) 111802 [arXiv:0708.1463] [INSPIRE].

[9] A. De Rujula et al., Higgs look-alikes at the LHC, Phys. Rev. D 82 (2010) 013003 [arXiv: 1001.5300] [INSPIRE].

[10] I. Low and J. Lykken, Revealing the electroweak properties of a new scalar resonance, JHEP 10 (2010) 053 [arXiv: 1005. 0872] [INSPIRE].

[11] H. Davoudiasl, T. McElmurry and A. Soni, Precocious diphoton signals of the little radion at hadron colliders, Phys. Rev. D 82 (2010) 115028 [Erratum ibid. D 86 (2012) 039907] [arXiv: 1009.0764] [INSPIRE].

[12] P.J. Fox, D. Tucker-Smith and N. Weiner, Higgs friends and counterfeits at hadron colliders, JHEP 06 (2011) 127 [arXiv:1104.5450] [InSPIRE].

[13] A. Strumia, Interpreting the $750 \mathrm{GeV}$ digamma excess: a review, in the proceedings of $51^{\text {st }}$ Rencontres de Moriond on Electroweak Interactions and Unified Theories, March 12-19, La Thuile, Italy (2016), arXiv: 1605.09401 [CERN-TH-2016-131].

[14] B.A. Dobrescu and F. Yu, Exotic signals of vectorlike quarks, J. Phys. G 45 (2018) 08LT01 [arXiv: 1612.01909] [INSPIRE].

[15] J.F. Gunion, H.E. Haber, G.L. Kane and S. Dawson, The Higgs hunter's guide, Front. Phys. 80 (2000) 1 [INSPIRE].

[16] D.S.M. Alves, P.J. Fox and N.J. Weiner, Higgs signals in a type I 2HDM or with a sister Higgs, arXiv:1207.5499 [INSPIRE]. 
[17] A.G. Akeroyd and M.A. Diaz, Searching for a light fermiophobic Higgs boson at the Tevatron, Phys. Rev. D 67 (2003) 095007 [hep-ph/0301203] [INSPIRE].

[18] A.G. Akeroyd, M.A. Diaz and F.J. Pacheco, Double fermiophobic Higgs boson production at the CERN LHC and LC, Phys. Rev. D 70 (2004) 075002 [hep-ph/0312231] [INSPIRE].

[19] A. Delgado et al., Diphoton and diboson probes of fermiophobic Higgs bosons at the LHC, JHEP 06 (2016) 042 [arXiv: 1603.00962] [INSPIRE].

[20] A. Arhrib et al., Identifying a light charged Higgs boson at the LHC Run II, Phys. Lett. B 774 (2017) 591 [arXiv: 1706.01964] [INSPIRE].

[21] U. Haisch and A. Malinauskas, Let there be light from a second light Higgs doublet, JHEP 03 (2018) 135 [arXiv: 1712.06599] [INSPIRE].

[22] G.C. Dorsch, S.J. Huber, K. Mimasu and J.M. No, Echoes of the electroweak phase transition: discovering a second Higgs doublet through $A_{0} \rightarrow Z H_{0}$, Phys. Rev. Lett. 113 (2014) 211802 [arXiv:1405.5537] [INSPIRE].

[23] N. Craig et al., The Hunt for the rest of the Higgs bosons, JHEP 06 (2015) 137 [arXiv: 1504.04630] [INSPIRE].

[24] D.S.M. Alves, S. El Hedri, A.M. Taki and N. Weiner, Charged Higgs signals in $t \bar{t} H$ searches, Phys. Rev. D 96 (2017) 075032 [arXiv:1703.06834] [INSPIRE].

[25] A. Djouadi, J. Kalinowski and P.M. Zerwas, Two and three-body decay modes of SUSY Higgs particles, Z. Phys. C 70 (1996) 435 [hep-ph/9511342] [INSPIRE].

[26] A.G. Akeroyd, Three body decays of Higgs bosons at LEP-2 and application to a hidden fermiophobic Higgs, Nucl. Phys. B 544 (1999) 557 [hep-ph/9806337] [INSPIRE].

[27] B. Coleppa, F. Kling and S. Su, Exotic decays of a heavy neutral Higgs through HZ/AZ channel, JHEP 09 (2014) 161 [arXiv:1404.1922] [INSPIRE].

[28] B. Coleppa, F. Kling and S. Su, Charged Higgs search via $A W^{ \pm} / H W^{ \pm}$channel, JHEP 12 (2014) 148 [arXiv: 1408.4119] [INSPIRE].

[29] F. Kling, A. Pyarelal and S. Su, Light charged Higgs bosons to AW/HW via top decay, JHEP 11 (2015) 051 [arXiv: 1504.06624] [INSPIRE].

[30] A. Arhrib, R. Benbrik and S. Moretti, Bosonic decays of charged Higgs bosons in a 2HDM type-I, Eur. Phys. J. C 77 (2017) 621 [arXiv:1607.02402] [INSPIRE].

[31] T. Enomoto and R. Watanabe, Flavor constraints on the two Higgs doublet models of $Z_{2}$ symmetric and aligned types, JHEP 05 (2016) 002 [arXiv:1511.05066] [INSPIRE].

[32] CMS collaboration, Search for charged Higgs bosons with the H+ to tau nu decay channel in the fully hadronic final state at $\sqrt{s}=8 \mathrm{TeV}$, CMS-PAS-HIG-14-020 (2014).

[33] CMS collaboration, Search for new resonances in the diphoton final state in the mass range between 70 and $110 \mathrm{GeV}$ in pp collisions at $\sqrt{s}=8$ and $13 \mathrm{TeV}$, CMS-PAS-HIG-17-013 (2017).

[34] ATLAS collaboration, Search for scalar diphoton resonances in the mass range 65-600 GeV with the ATLAS detector in pp collision data at $\sqrt{s}=8, T e V$, Phys. Rev. Lett. 113 (2014) 171801 [arXiv: 1407.6583 ] [INSPIRE].

[35] CMS collaboration, Search for associated production of Higgs bosons and top quarks in multilepton final states at $\sqrt{s}=13 \mathrm{TeV}$, CMS-PAS-HIG-16-022 (2016). 
[36] ATLAS collaboration, Search for the associated production of a Higgs boson and a top quark pair in multilepton final states with the ATLAS detector, ATLAS-CONF-2016-058 (2016).

[37] CMS Collaboration, Search for $t \bar{t} H$ production in the $H \rightarrow b \bar{b}$ decay channel with $2016 p p$ collision data at $\sqrt{s}=13 \mathrm{TeV}$, CMS-PAS-HIG-16-038 (2016).

[38] ATLAS collaboration, Measurement of fiducial, differential and production cross sections in the $H \rightarrow \gamma \gamma$ decay channel with $13.3 \mathrm{fb}^{-1}$ of $13 \mathrm{TeV}$ proton-proton collision data with the ATLAS detector, ATLAS-CONF-2016-067 (2016).

[39] CMS collaboration, Search for Higgs boson production in association with top quarks in multilepton final states at $\sqrt{s}=13 \mathrm{TeV}$, CMS-PAS-HIG-17-004 (2017).

[40] CMS collaboration, Updated measurements of Higgs boson production in the diphoton decay channel at $\sqrt{s}=13 \mathrm{TeV}$ in pp collisions at CMS, CMS-PAS-HIG-16-020 (2016).

[41] CDF collaboration, T. Aaltonen et al., Search for the standard model Higgs boson produced in association with top quarks using the full CDF data set, Phys. Rev. Lett. 109 (2012) 181802 [arXiv: 1208.2662] [INSPIRE].

[42] CDF collaboration, T. Aaltonen et al., Combination of searches for the higgs boson using the full CDF data set, Phys. Rev. D 88 (2013) 052013 [arXiv: 1301.6668] [INSPIRE].

[43] G. Cacciapaglia et al., Search for a lighter Higgs boson in two Higgs doublet models, JHEP 12 (2016) 068 [arXiv: 1607.08653] [INSPIRE]. 